\title{
Geometry and Profile Modification of Microcantilevers for Sensitivity Enhancement in Sensing Applications
}

\author{
Yan Liu, ${ }^{1,2}$ Hai Wang, ${ }^{1 *}$ Hongbo Qin, ${ }^{1}$ Wei Zhao, ${ }^{1}$ and Peng Wang ${ }^{2}$ \\ ${ }^{1}$ Key Laboratory of Electronic Equipment Structure Design, Ministry of Education, \\ School of Electro-Mechanical Engineering, Xidian University, Xi'an 710071, China \\ ${ }^{2}$ State Key Laboratory for Manufacturing Systems Engineering, Xi'an Jiaotong University, Xi'an 710054, China
}

(Received September 8, 2016; accepted December 16, 2016)

Keywords: microcantilever, resonant frequency, geometry modification

The issues of absorbed-gas-induced frequency shift of a microcantilever with various geometries and profiles are investigated in this paper to enhance the measurement sensitivity of a microcantilever-based gas sensor. The resonant frequency of microcantilevers with different shapes is investigated by the analytical method and finite element method. Considering the dependence of frequency shift on the mass distribution of absorbed gas and structural resonant frequency, a principle is established for increasing the measurement sensitivity when the target gas is absorbed. Using these results, a stepped microcantilever with a trapezoidal-shaped part at the clamped end and a rectangular pad at the free end, which is expected to have better performance, is proposed as a sensing element in gas detection.

\section{Introduction}

Microcantilevers have been demonstrated to be extremely versatile sensors utilizing mechanical, optical, electrostatic or electromagnetic methods ${ }^{(1,2)}$ of sensing the changes in cantilever mechanical properties to gain insight into gas concentration, biomolecular interactions or surface topography. ${ }^{(3-5)}$ Microcantilever-based sensors for gas detection usually use a functional material coating layer to capture the molecules of a target stimulus from the ambient environment. The sorption of gases induces the modification of the physicochemical characteristics of a coating material, and consequently, the mechanical properties of the microstructure. Two experimental modes exist to identify these changed parameters; one is the static mode that measures the surfacestress-induced deflection of the microcantilever, and the other is the dynamic mode that measures the shift of the resonant frequency due to the added mass. ${ }^{(6,7)}$

Since Stoney's formula serves as a widely accepted basal function linking the surface stress and the radius of curvature in bent cantilevers, ${ }^{(8)}$ several groups have accomplished significant work in improving the device sensitivity and experimental accuracy of deflection-based microcantilevers. However, the static deflection-based measurement is generally conducted in well-controlled laboratory environments to ensure experimental accuracy, and the necessary calibration process can be error-prone and time-consuming. ${ }^{(9)}$ Moreover, the deflection and resonant frequency, which are two restrictive parameters, have to be enhanced at the same time when the deflection-based microcantilever is designed.

${ }^{*}$ Corresponding author: e-mail: wanghai@mail.xidian.edu.cn

http://dx.doi.org/10.18494/SAM.2017.1465 
The dynamic sensing mode, dispensing with the deflection calibration procedure or trade-off between restrictive parameters, may be useful in many applications. In the dynamic sensing mode, the resonant frequency is a vital parameter for microcantilever-based sensors. High-frequency sensitivity can be obtained in systems with high resonant frequency. Many models have been developed to optimize the performance for the requirements of sensing applications by changing the beam size, material, geometry or profile. Minimizing the cantilever, modifying the geometry, and improving the cantilever profiles are all valid approaches. However, the microcantilevers cannot be arbitrarily modified owing to the restrictions from fabrication and active region on the surface of microcantilevers. The active sensing area proportionately determines the absorptioninduced variation of mass for gas sensors, influencing the frequency sensitivity. Therefore, the modification via focused ion beam milling reported in Ref. 10 is not a very effective way of achieving high-frequency microcantilever-based sensors. Zhang et al. ${ }^{(11)}$ obtained the fundamental resonant frequencies of microcantilevers with various linear geometries by using the variational method but did not consider the effect of the active sensing area. The frequency properties of microcantilevers with varying profiles were also investigated by Fernando et al. ${ }^{(12)}$ The very essence of modifying the geometry or profile is to improve the weight distribution of microcantilevers, which is a beneficial scheme for microcantilever design. However, little work that discusses the improvement of the microcantilever weight distribution by changing both the geometry and profile of the microcantilever has been reported to the best of our knowledge.

In this paper, the gas-adsorption-induced resonant frequency shift of a microcantilever with various geometries and profiles and the sensitivity promotion via shape modification for microcantilever-based sensors are investigated. After introducing the sensing principle, the RayleighRitz (R-R) method is utilized to formulate the resonant frequencies of prismatic and nonprismatic microcantilevers. The explicit formulas, simple and convenient for application, are validated by the finite element method (FEM) simulation. Moreover, the dependence of sensitivity on the location of the sensing region is also considered. Using these results, the effects of microcantilever shape and sensing region parameters on the measurement sensitivity are studied and a design guideline for promoting the sensor performance via microcantilever structural modification is presented. On the basis of this guideline, an efficacious microcantilever structure is proposed for gas sensing applications.

\section{Theories}

\subsection{Sensing principle of dynamic microcantilever-based gas sensor}

When a microcantilever with a sensitive coating layer is placed in an environment in the presence of a target gas, the resonant frequency shift of the cantilever induced by sorption is expressed as ${ }^{(13)}$

$$
\Delta f=-\frac{1}{2} \frac{\Delta m}{m_{e f f}} f_{0},
$$

assuming that the variation of the stiffness caused by added mass is generally negligible. In Eq. (1), $\Delta f$ is the resonant frequency shift, $\Delta m$ is the added mass, $m_{\text {eff }}$ is the cantilever effective mass, and $f_{0}$ is the resonant frequency. Considering the partition coefficient $K$ of the sensitive coating for the 
considered gas, the added mass can be obtained as ${ }^{(13)}$

$$
\Delta m=K h_{\Delta} \Sigma C_{g},
$$

where $h_{\Delta}$ is the thickness of the sensitive layer, $\Sigma$ is the area of the sensitive region, and $C_{g}$ is the gas concentration.

Let

$$
A=K h_{\Delta} C_{g}
$$

Equation (1) can be rewritten as

$$
\Delta f=-\frac{A}{2} \frac{\Sigma}{m_{\text {eff }}} f_{0} .
$$

$A$ indicates the parameters that have no relationship with the microcantilever geometry or profile. For a greater shift in frequency, the values of $f_{0}$ and $\Sigma$ should be very large and the effective mass of the cantilever should be minimized. $f_{0}$ and $m_{\text {eff }}$ can be adjusted by changing the weight distribution of the cantilever, which can be achieved by modifying the cantilever geometry and profile. Meanwhile, the determination of $\Sigma$ is not only related to the cantilever shape, but also to the tradeoff with the area for the signal transduction elements in the sensor.

\subsection{Resonant frequency of cantilevers with varying geometries and profiles}

Figure 1 shows the structure of the microcantilever with a linear boundary, whose resonant frequency can be formulated by the R-R method. ${ }^{(14)}$ Using the structural deflection function $W(x)$, the kinetic energy $T$ of the cantilever can be expressed as

$$
T=\frac{1}{2} \omega^{2} \iiint_{V} W(x)^{2} d m
$$

where $d m$ is the mass of elementary volume and $\omega$ is the angular frequency. The potential energy $U$ of the structure is

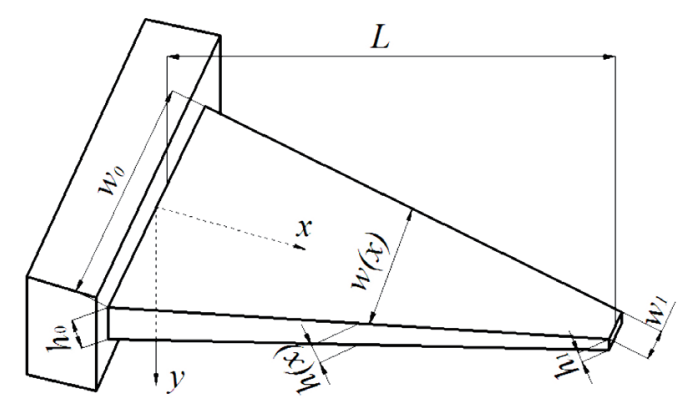

Fig. 1. Schematic of a microcantilever with varying geometry and profile. 


$$
U=\frac{1}{2} \int_{L} E I\left(\frac{d^{2} W(x)}{d x^{2}}\right) d x,
$$

where $E$ is Young's modulus, and $I$ is the cross-sectional area moment of inertia. According to the conservation law of mechanical energy, the kinetic energy is equal to the potential energy. Then, the resonant frequency of the microcantilever can be defined as

$$
f_{0}=\frac{\omega}{2 \pi}=\frac{1}{2 \pi} \sqrt{\frac{\int_{L} E I\left(d^{2} W(x) / d x^{2}\right) d x}{\iiint_{V} W(x)^{2} d m}} .
$$

Let

$$
u=w_{0}-w_{1}, v=h_{0}-h_{1},
$$

where $w_{0}$ and $w_{1}$ are the widths of the clamped and free ends, and $h_{0}$ and $h_{1}$ are the thicknesses of the clamped and free ends, respectively. The cantilever width $w(x)$ and the thickness $h(x)$ are functions of the distance from the clamped end $x$.

For a prismatic microcantilever with varying geometry, $v=0$ and $w(x)=w_{0}-u x / L$. The following expression is obtained for its resonant frequency:

$$
f_{0-p}=\frac{h_{0}}{2 \pi} \sqrt{\frac{3 E}{\rho_{1}}} \sqrt{\frac{\int_{0}^{L} w(x)(L-x)^{2} d x}{\int_{0}^{L} w(x) x^{4}(3 L-x)^{2} d x}}=\frac{1}{2 \pi} \sqrt{\frac{3 E}{\rho_{1}}} \frac{h_{0}}{L^{2}} \sqrt{\frac{210+70 w_{1} / w_{0}}{147+645 w_{1} / w_{0}}},
$$

where $\rho_{1}$ is the density of the cantilever material. Then, the resonant frequency of rectangular microcantilevers with a varying profile, $u=0, h(x)=h_{0}-v x / L$, is considered. The formulation for its natural frequency can be derived as

$$
f_{0-r}=\frac{1}{2 \pi} \sqrt{\frac{3 E}{\rho_{1}}} \frac{1}{L^{2}} \sqrt{\frac{280 h_{0}^{3}-210 h_{0}^{2} v+84 h_{0} v^{2}-14 v^{3}}{792 h_{0}-645 v}} .
$$

As we all know, the R-R method's accuracy depends on the correctness of the deflection function. If the deflection function matches the actual mode of vibration well, the calculated frequency will highly approximate the actual resonant frequency. On the other hand, if the assumed function cannot perfectly represent the true vibration mode, the analytical frequency from the R-R method will be higher than the real value because the inappropriate deflection mode applies added restrictions to the beam. For a nonprismatic microcantilever with varying geometry, it is difficult to obtain the function for its deflection, and the R-R method cannot be carried out without an accurate deflection formulation. In order to simplify the work, FEM will be used to investigate the resonant frequency of the nonprismatic microcantilevers with varying geometries.

When the sensitive region entirely occupies the upper surface of the cantilever, the effective mass of the prismatic cantilever can be expressed as 


$$
m_{e f f}=N \Sigma h_{0} \rho_{1},
$$

where $N$ is a parameter that depends on the geometry of the cantilever. By substituting Eqs. (9) and (11) into Eq. (4), the frequency shift expressed by Eq. (4) can be rearranged as

$$
\Delta f_{0_{-} p}=\frac{-A}{4 \pi N \rho_{1}} \sqrt{\frac{3 E}{\rho_{1}}} \frac{1}{L^{2}} \sqrt{\frac{210+70 w_{1} / w_{0}}{147+645 w_{1} / w_{0}}} .
$$

As seen from Eq. (12), the frequency shift is determined by the cantilever length and the ratio of the free end width to the clamped end width when the prismatic cantilever is used in a certain experimental environment. This width ratio $w_{1} / w_{0}$ reflects the cantilever weight distribution along the length direction and its decrease enhances the frequency sensitivity. The change in cantilever length affects the cantilever volume and spring constant, generating a marked variation in the sensor sensitivity. However, it is not advantageous to extremely shorten the cantilevers as it reduces the cantilever deflection considerably, making it difficult to detect the vibration. Thickness is another parameter that affects the cantilever frequency whose increase makes the frequency acutely increase, but thickness has no effect on the frequency shift of the prismatic cantilever according to Eq. (12). Thus, it is beneficial for the prismatic microcantilevers to reduce the mass near the free end by increasing the taper ratio. For a rectangular microcantilever with varying profile, the same principle is also valid.

\subsection{Dependence of sensitivity on position of sensing region}

The mass-induced change in resonant frequency is not only dependent on the mass of the absorbed particles, but also on the position of particles in the cantilever, especially when the target gas concentration is low. The regions with a large vibrational amplitude are those where the absorbed particles can gain a high kinetic energy and thereby change the resonant frequency considerably when compared with the ones where little energy transfer happens. Therefore, the sensing region should be located near the free end of the cantilever to promote the sensitivity when designing the cantilever for low-concentration gas detection.

\section{Results and Discussion}

In this section, the validities of equations for calculating the resonant frequencies of microcantilevers with various geometries and profiles are investigated. Simulations are carried out using the finite element software ANSYS Multiphysics, and the analytical values are verified by FEM results. The relationship between measurement sensitivity and microcantilever shape are investigated and a design guideline for structural modification is presented. Then, an available optimized structural scheme for a microcantilever-based gas sensor is proposed. In the FEM simulations, all the three-dimensional models for different cantilevers are constructed and analyzed using the APDL language. The element type is set as SOLID 95, a kind of 20-node solid element defined in the software, and all degrees of freedom of the fixed end in cantilevers are locked when conducting the modal analyses by ANSYS Multiphysics. The material properties used in this section are $130 \mathrm{GPa}$ for Young's modulus, $2330 \mathrm{kgm}^{-3}$ for density, and 0.27 for Poisson's ratio. The 
gas absorption in the microcantilever is a very complex process, which is very difficult to simulate. Meanwhile, in this paper, we mainly focus on the mass-variation-induced frequency shift during gas absorption, and the gas type and corresponding functional layer are not very related to the cantilever structural design. Then, the added mass induced by gas absorption is simulated by changing the density of the material in the sensing region from 2330 to $2331 \mathrm{kgm}^{-3}$.

\subsection{Resonant frequency of microcantilevers}

For prismatic microcantilevers, the resonant frequency is increased by reducing the ratio of the free end width to the clamped end width according to Eq. (9). The decrease in cantilever length and increase in thickness can lead to an increase in the resonant frequency. These changing trends can also be found in Fig. 2. The results obtained from the R-R method and FEM show a remarkable resemblance, and the maximum differences are 4.39, 2.33, and 4.06\% for $L=350$, 300 , and $250 \mu \mathrm{m}$ with $h_{0}=1 \mu \mathrm{m}$, and 3.36, 2.33, and $1.61 \%$ for $h_{0}=0.5,1$, and $1.5 \mu \mathrm{m}$ with $L$ $=300 \mu \mathrm{m}$, respectively. The triangular microcantilever $\left(w_{1} / w_{0}=0\right)$ gives a twofold increase in the resonant frequency compared with the rectangular microcantilever. Clearly, the triangular microcantilever has better potential in the sensing applications.

Figure 3 shows $f_{0}$ versus $h_{1} / h_{0}$ with $h_{0}=1,1.5$, and $2 \mu \mathrm{m}$ when $w_{0}=w_{1}=80 \mu \mathrm{m}$, and $L=$ $300 \mu \mathrm{m}$. The reduction in $h_{1} / h_{0}$ contributes much towards the resonant frequency. From Fig. 3, it is seen that for $h_{1} / h_{0}=1$ when $h_{0}=1 \mu \mathrm{m}$, and $L=300 \mu \mathrm{m}$, the resonant frequency, taking the analytical results for instance, is $13.6 \mathrm{kHz}$, and the value becomes $20.5 \mathrm{kHz}$ when $h_{1} / h_{0}=0.05$, which is an improvement of 50.9\%. The trend in Fig. 3 also indicates that an increase in the thickness of the clamped end leads to an enhancement in the resonant frequency. The analytical results have larger errors when the free end is thin. The maximum errors in Fig. 3 are 8.57, 8.61, and $8.63 \%$ for $h_{0}=1,1.5$, and $2 \mu \mathrm{m}$, respectively. The mismatch between the formulated and simulated deflections may contribute to these large frequency differences. The deflection formula used in the R-R method is derived from the deflection of the standard rectangular microcantilever

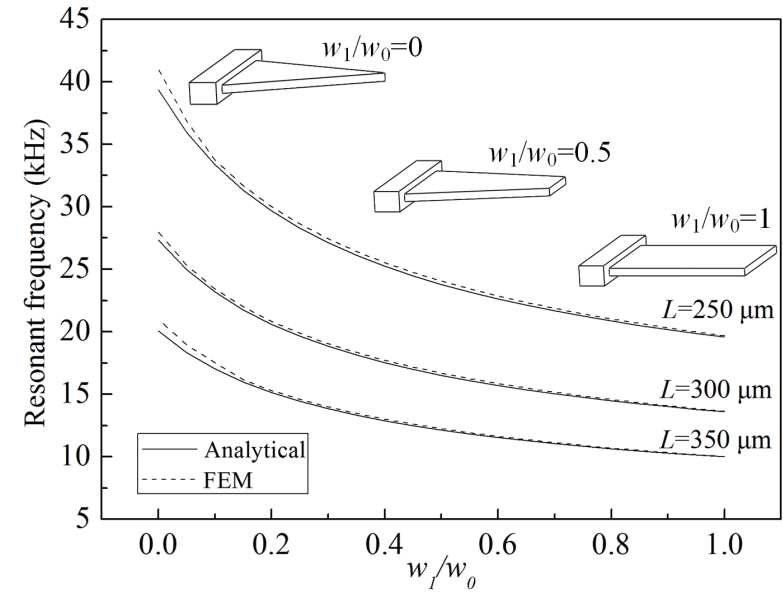

(a)

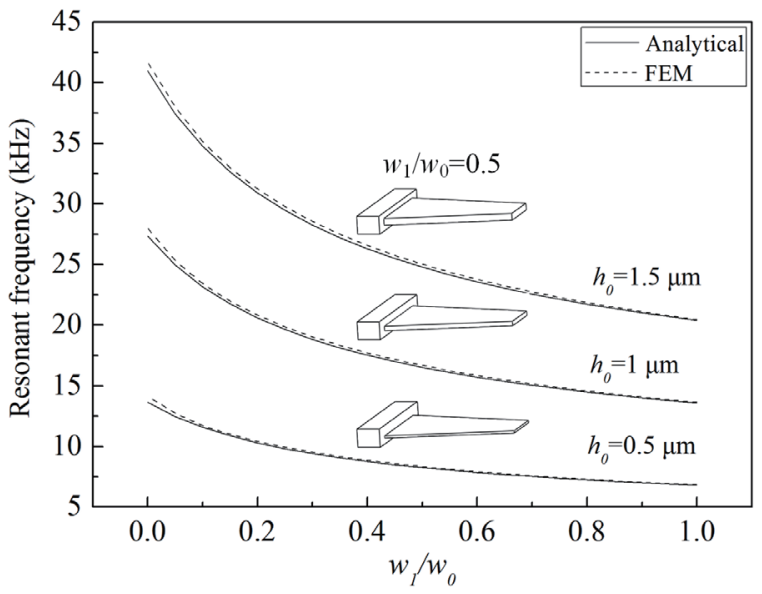

(b)

Fig. 2. Resonant frequency for prismatic microcantilevers with varying geometries, (a) for $L=350,300$, and 250 $\mu \mathrm{m}$ with $h_{0}=1 \mu \mathrm{m}$ and (b) for $h_{0}=0.5,1$, and $1.5 \mu \mathrm{m}$ with $L=300 \mu \mathrm{m}$. 


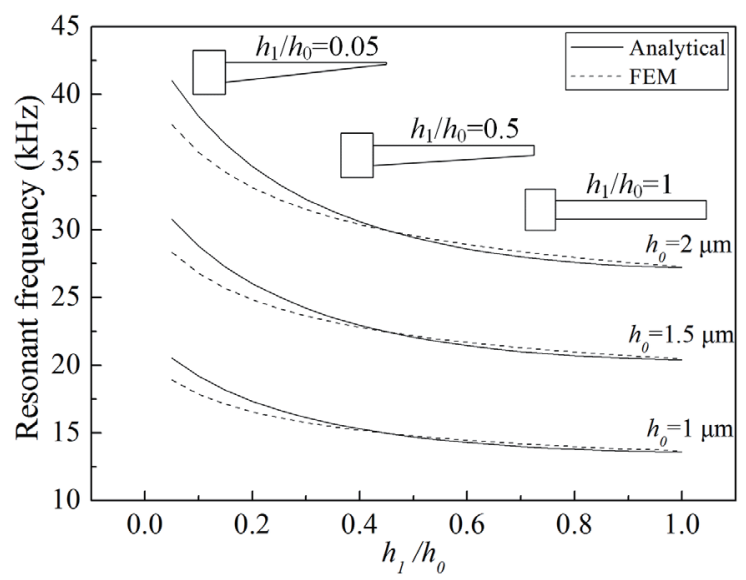

Fig. 3. Resonant frequency versus $h_{1} / h_{0}$ with $h_{0}=1,1.5$, and $2 \mu \mathrm{m}$ when $w_{0}=w_{1}=80 \mu \mathrm{m}, L=300 \mu \mathrm{m}$.

with a uniform section, but when the ratio $h_{1} / h_{0}$ varies, the deformation of the microcantilever becomes more complex, not complying anymore with the deflection of a standard rectangular microcantilever. This deflection mismatch gives extra restrictions to the cantilever, increasing the analytical frequency.

It can be seen from the above results that upon reducing the width or thickness of the free end, the resonant frequency of the cantilever is enhanced. Meanwhile, the improvement can be upgraded. Figure 4 shows the results from simulations of the microcantilevers with various geometries and profiles. To avoid confusion, the surface area on the top of the proposed microcantilevers, namely, the area of the available active sensing region, is kept constant, the same with the rectangular microcantilever with $80 \mu \mathrm{m}$ width and $300 \mu \mathrm{m}$ length. The resonant frequency is greatly increased by geometry and profile modifications. The maximum value is 31.8 $\mathrm{kHz}$, appearing at $w_{1} / w_{0}=0$ and $h_{1} / h_{0}=0.1$, which is increased by $233 \%$ compared with the value at $w_{1} / w_{0}=1$ and $h_{1} / h_{0}=1$. In reality, the maximum frequency appears under the condition of $h_{1} / h_{0}=0$, but the zero thickness cannot be obtained in the microcantilever fabrication. Herein, the minimum thickness of the free end is set as $10 \%$ of the clamped end thickness.

\subsection{Sensitivity and structural optimization}

With the resonant frequency results for cantilevers, the sensor sensitivity is further investigated, and a series of microcantilevers are molded and simulated using FEM software. Figure 5 shows the frequency shift and mass sensitivity versus the width of the free end, $w_{1}$, with $L=250,300$, and $350 \mu \mathrm{m}$ when $w_{0}=100 \mu \mathrm{m}, h_{0}=h_{1}=1.5 \mu \mathrm{m}$. Herein, the added mass is assumed to be absorbed uniformly over the entire cantilever surface and changes with the geometry. The same with the resonant frequency, the reduction of $L$ also contributes much towards the frequency shift and sensitivity. The enhancement can be up to $200 \%$ for frequency shift and $280 \%$ for sensitivity when $L$ changes from 350 to $250 \mu \mathrm{m}$ at $w_{1}=0 \mu \mathrm{m}$. Meanwhile, along with the variation of $\mathrm{w}_{1}$, the area of the cantilever surface also exhibits a clear diversity, leading to a decrease in the total mass that the cantilever can absorb, which may decrease the sensing threshold of gas concentration. To eliminate the influence of sensing region reduction, a further study is proposed with different combinations of $w_{0}, L$, and $w_{1}$, but with a constant sensing area. For certain $w_{0}$ and $w_{1}$, the cantilever length $L$ is 


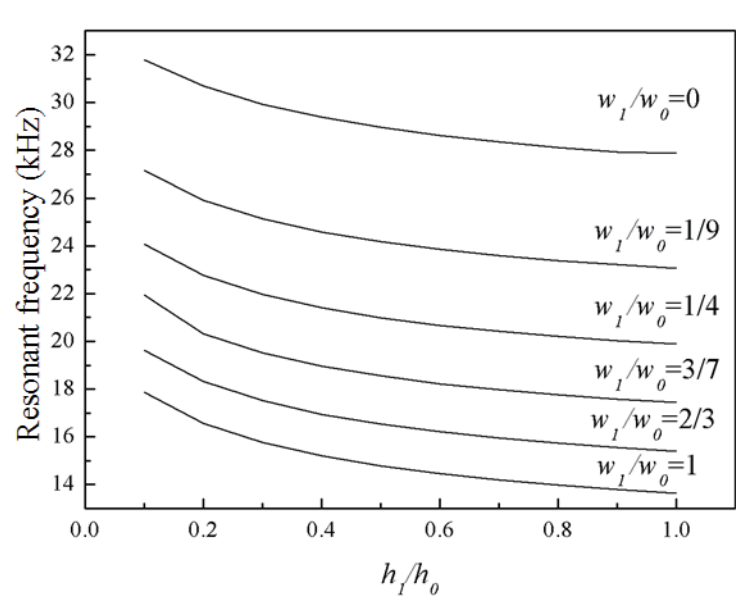

Fig. 4. Resonant frequency from FEM for nonprismatic microcantilevers with varying geometries whose areas of the sensing region are constant.

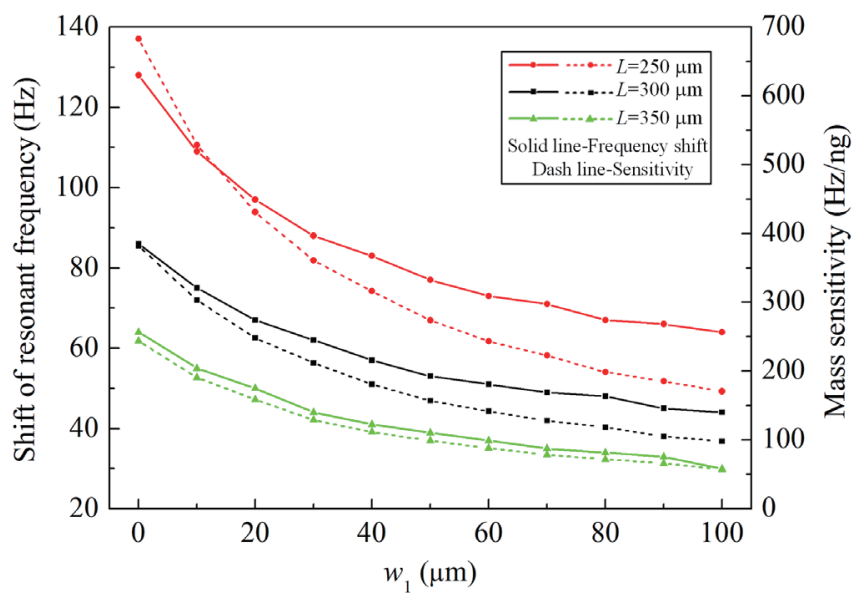

Fig. 5. (Color online) Shift of resonant frequency and sensitivity versus $w_{1}$ with $L=250,300$, and 350 $\mu \mathrm{m}$ when $w_{0}=100 \mu \mathrm{m}, h_{0}=h_{1}=1.5 \mu \mathrm{m}$.

accordingly calculated to obtain the same sensing area as the cantilever with $w_{0}=w_{1}=100 \mu \mathrm{m}$, $L=300 \mu \mathrm{m}$. The grouped dimensions are simply outlined in Fig. 6 . The mass sensitivity can be considerably promoted by widening the clamped end and shortening the cantilever. Changing $w_{1}$ will no longer be an effective proposal in this situation, because the variation in $w_{1}$ will induce a change in $L$ to maintain a constant sensing area.

As mentioned in Sect. 2.3, the location of added mass also plays a very important role in the sensor measurement sensitivity. Figure 7 shows the position influence on the frequency shift or measurement sensitivity in a rectangular cantilever sensor with $w_{0}=w_{1}=100 \mu \mathrm{m}, L=300 \mu \mathrm{m}$, $h_{0}=h_{1}=2.45 \mu \mathrm{m}$, in which the sensing area occupies half of the cantilever upper surface. The frequency shift is promoted by about 18 times by moving the sensing region from the clamped end to the free end, denoting the clear effect on sensor performance from the mass position. The enhancement can also be obtained in the triangular cantilever at a more distinguished level.

Considering the abovementioned results, it is clear that heightening the resonant frequency and locating the added mass near the free end will significantly enhance the microcantilever sensitivity. Heightening the resonant frequency can be realized by shortening the cantilever, widening the clamped end, and minimizing the effective mass near the microcantilever's free end. Meanwhile, locating the added mass near the free end requires that the large sensing region is arranged close to the free end. The restrictive relationship between the resonant frequency promotion and the arrangement of the sensing region should be relieved when optimizing the cantilever structure. To mitigate this issue, an available scheme is proposed, which is a stepped microcantilever with a trapezoidal-shaped part at the clamped end and a rectangular pad at the free end, as shown in Fig. 8. In the optimized structure, the thick trapezoidal-shaped part possesses a wide clamped end, maintaining the high stiffness of the cantilever; the thin rectangular pad, with the sensing region above its surface, can reduce the effective mass near the free end and locate the added mass at the free end simultaneously. Relational simulation for the proposed cantilever is conducted, and the results are listed and compared in Table 1. In the simulations, the length, clamped end thickness, 


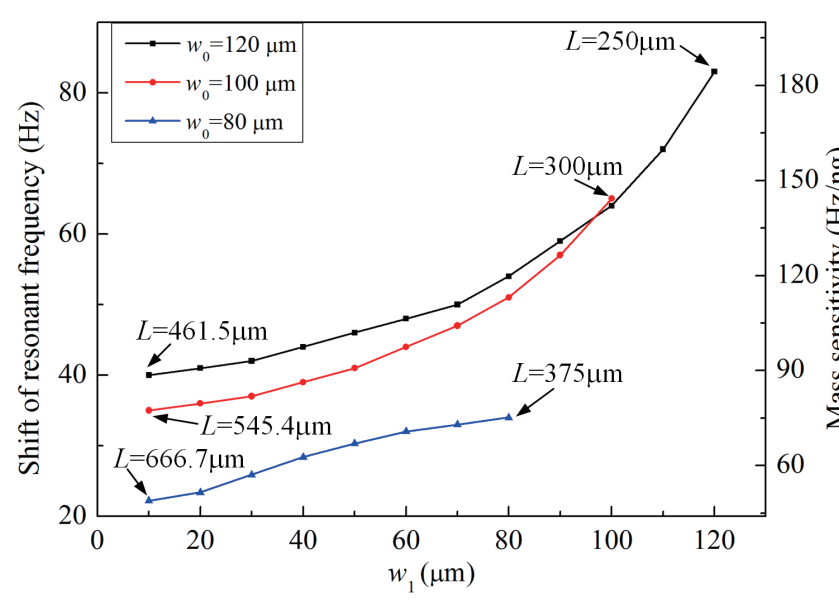

Fig. 6. (Color online) Shift of resonant frequency and sensitivity versus $w_{1}$ with $w_{0}=80,100$, and 120 $\mu \mathrm{m}$ when the area of the cantilever surface is kept constant.

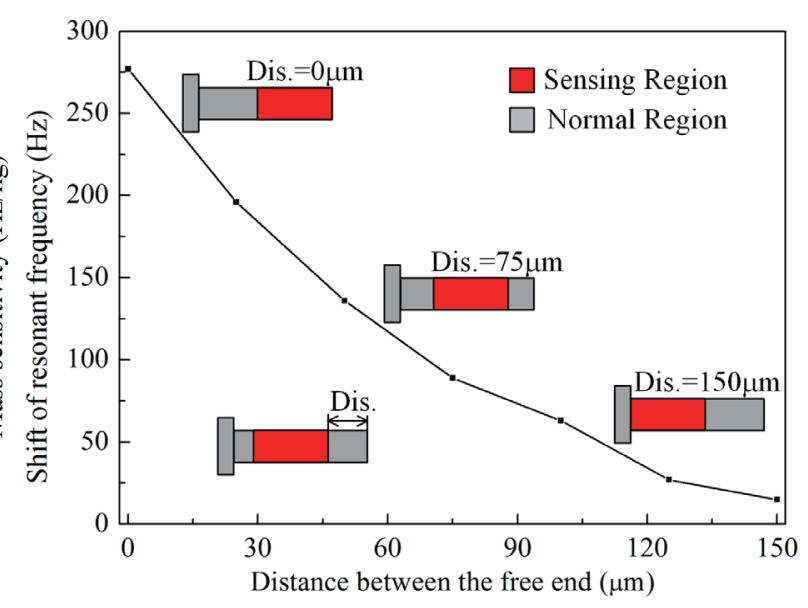

Fig. 7. (Color online) Position dependence of the rectangular cantilever sensitivity.

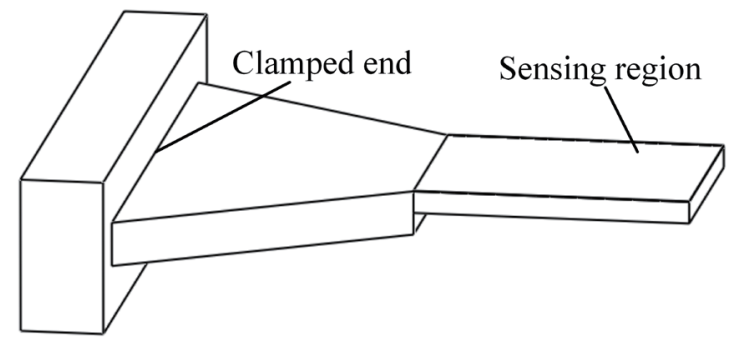

Fig. 8. Optimized stepped cantilever for sensitivity enhancement.

Table 1

Comparison between the optimized and normal structures.

\begin{tabular}{lcccc}
\hline Structure & $\Delta f_{0}$ & $\Delta m$ & Sensitivity \\
\hline Rectangular cantilever & 1 & 1 & 1 \\
Stepped rectangular cantilever & 2.81 & 1 & 2.81 \\
Optimized stepped cantilever & 3.16 & 1 & 3.16
\end{tabular}

and area of the sensing region are all kept constant. The thickness of the free end is determined according to the cantilever profile. The width of the clamped end in the optimized scheme is two times that in the rectangular geometry. The results are all normalized with reference to the characteristics of the normal rectangular cantilever. Compared with the normal rectangular cantilever, the optimized structure increases the resonant frequency or measurement sensitivity by about $316 \%$, obtaining a clear enhancement in the sensor performance. 


\section{Conclusions}

In this paper, the resonant frequencies of microcantilevers are formulated and analyzed by the R-R method and FEM simulation. The explicit analytical formulas are validated by the FEM simulation, and the maximum difference between the two methods is $8.63 \%$, which is induced by the extra constraints from analytical deflections. It is demonstrated that the microcantilevers with a minimum mass near the free end can provide a larger frequency shift when the target gas is absorbed, which can be achieved by making the free end of the cantilever thinner and narrower than the clamped end. Meantime, arranging the sensing region near the free end can also enhance the sensor performance. A novel stepped microcantilever with a trapezoidal-shaped part at the clamped end and a rectangular pad at the free end is developed to minimize the cantilever mass near its free end and maintain a sufficient area for gas sensing to locate more absorbed gas near the free end. The results show that the improved microcantilever exhibits a more excellent measurement sensitivity, which is more than three times that of the simple rectangular-shaped prismatic microcantilever.

\section{Acknowledgments}

This research was supported by the National Natural Science Foundation of China (No. 51505358), the Fundamental Research Funds for the Central Universities of China (No. JB160409), and the Open Foundation of the State Key Laboratory for Manufacturing Systems Engineering (No. sklms201511).

\section{References}

1 Y. Liu, H. Wang, H. Qin, W. Zhao, and P. Wang: Proc. 2016 Int. Symp. Computer, Consumer and Control (IEEE, Xi'an, 2016) p. 610.

2 P. S. Waggoner and H. G. Craighead: Lab Chip 7 (2007) 1238.

3 J. El-Ali, P. K. Sorger, and K. F. Jensen: Nature 442 (2006) 403.

4 J. Fritz: Analyst 133 (2008) 855.

5 S. H. Wang, S. L. Tan, G. Xu, and K. Koyama: Meas. Sci. Technol. 22 (2011) 094013.

6 P. Lu, H. P. Lee, C. Lu, and S. J. O’Shea: Phys. Rev. B 72 (2005) 085405.

7 J. E. Sader: J. Appl. Phys. 89 (2001) 2911.

8 G. G. Stoney: Proc. R. Soc. London, Ser. A 82 (1909) 172.

9 A. W. McFarland, M. A. Poggi, M. J. Doyle, L. A. Bottomley, and J. S. Colton: Appl. Phys. Lett. 87 (2005) 053505.

10 A. R. Hodges, K. M. Bussmann, and J. H. Hoh: Rev. Sci. Instrum. 72 (2001) 3880.

11 G. Zhang, L. Zhao, Z. Jiang, S. Yang, Y. Zhao, E. Huang, R. Hebibul, X. Wang, and Z. Liu: J. Phys. D: Appl. Phys. 44 (2011) 425402.

12 S. Fernando, M. Austin, and J. Chaffey: J. Phys. D: Appl. Phys. 40 (2007) 7652.

13 L. Fadel, I. Dufour, F. Lochon, and O. Francais: Sens. Actuators, B 102 (2004) 73.

14 G. Rinaldi, M. Packirisamy, I. Stiharu, and N. Mrad: Sens. Rev. 29 (2009) 44. 